\title{
Reduction of carbon dioxide by a molybdenum-containing formate dehydrogenase: a kinetic and mechanistic study
}

\author{
Luisa B. Maia, Luis Fonseca, Isabel Moura and José J.G. Moura \\ UCIBIO, REQUIMTE, Departamento de Química, Faculdade de Ciências e Tecnologia, Universidade Nova \\ de Lisboa, 2829-516 Caparica, Portugal.
}

\section{SUPPORTING INFORMATION}

\section{Index:}

S1. Figures S1 to S4 page S2

S2. Kinetic model for the FDH-catalyzed formate oxidation and carbon dioxide reduction page 56

S3. FDH reductive activation page $\mathrm{S} 8$

S4. The sulfur shift mechanism (including figure S5) page $\mathrm{S} 10$

S5. Selenocysteine dissociation from the FDH molybdenum center and formate binding to molybdenum

page $\mathrm{S} 13$

S6. EPR spectroscopic studies of FDH page S16

S7. Rate equations $\mathrm{S} 1$ to $\mathrm{S} 3$ page S18

References (the references cited only in this document are numbered as S\# and listed in the end) 


\section{S1. Figures}

\section{A (E.coli FDH H oxid.)}

B (E.coli FDH H reduced)

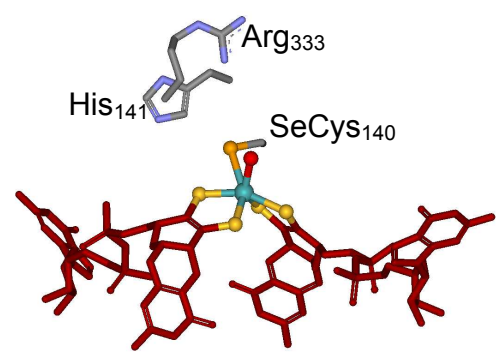

D (E.coli FDH N)

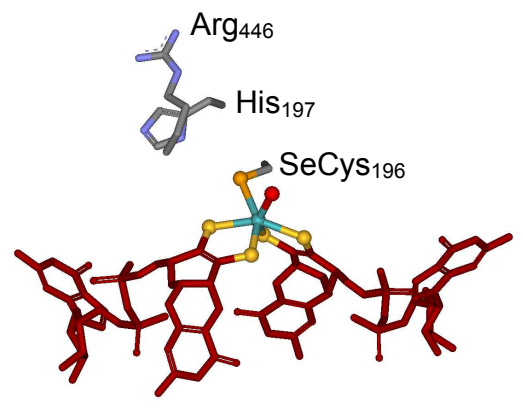

$\operatorname{Arg}_{333}$

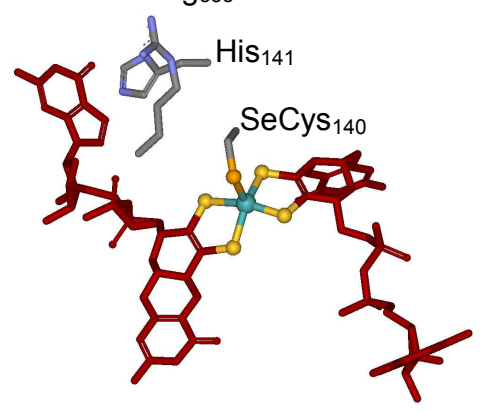

C (E.coli FDH H reduced reinterpretation)

His 141

SeCys 140

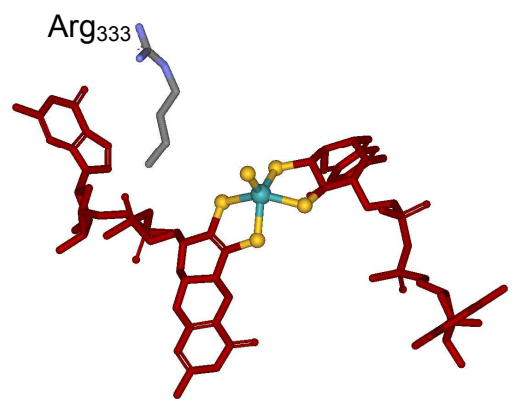

E (D. gigas $\mathrm{FDH})$

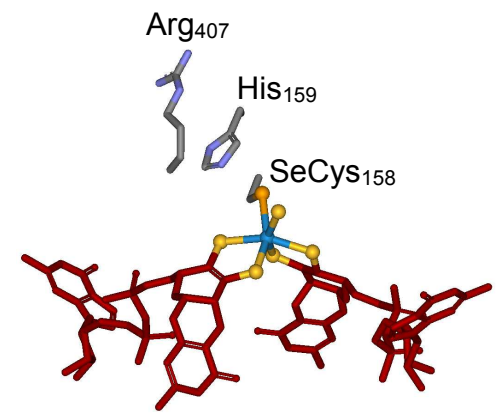

Figure S1. The active site of formate dehydrogenases. Three-dimensional structure view of the oxidized E. coli $\mathrm{FDH} \mathrm{H}$ catalytic center (panel A), with the molybdenum atom coordinated by a conserved essential selenocysteine residue and a sulfo terminal group, in a distorted trigonal prismatic coordination geometry. ${ }^{10}$ In the reduced state, the molybdenum center of $E$. coli $\mathrm{FDH} \mathrm{H}$ displayed a distorted square-pyramidal geometry; Boyington et al. ${ }^{10}$ identified the apical ligand as the selenium atom of the selenocysteine residue (panel B), but Raaijmakers and Romão ${ }^{11}$, in a posterior reinterpretation of the original data, demonstrated that the apical position is occupied by a sulfur atom and that the selenocysteine residue is shifted away $(12 \AA)$ from the molybdenum center (panel $\mathbf{C}$ ). The three-dimensional structure view of the oxidized catalytic center of $E$. coli Mo-FDH N ${ }^{12}$ and of D. gigas W-FDH ${ }^{8 f}$ is represented in panels $\mathbf{D}$ and $\mathbf{E}$, respectively. The pyranopterin cofactor (Fig. 1.C) is represented in dark red. The structures shown are based on the PDB files 1FDO (A), 1AA6 (B), 2IV2 $(C), 1 \mathrm{KQF}(\mathrm{D})$ and $1 \mathrm{HOH}(\mathrm{E})$. The images were produced with Accelrys DS Visualizer and Accelrys Draw (Accelrys Software Inc). 


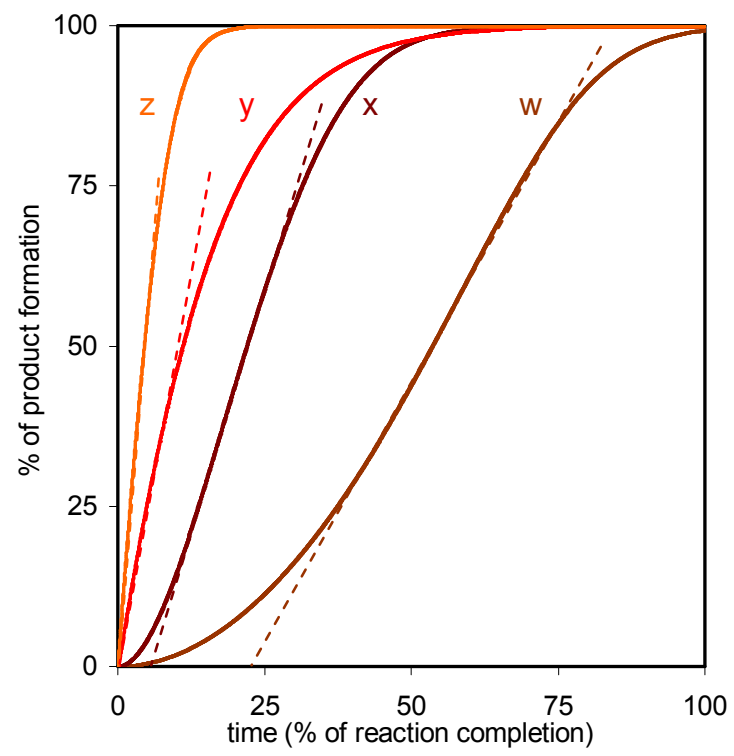

Figure S2. Simulated time courses of Dd FDH-catalyzed formate oxidation. Curves (w) to (z) are simulations based on the kinetic model presented in figure 4 , using the following $k_{i}$ values (in arbitrary values): $k_{5}=1 \mathrm{M}^{-1} \mathrm{~s}^{-1}, k_{6}=0.001 \mathrm{~s}^{-1}, k_{6}=0.0001 \mathrm{~s}^{-1}(\mathrm{w}), k_{5}=1 \mathrm{M}^{-1} \mathrm{~s}^{-1}, k_{6}=0.01 \mathrm{~s}^{-1}$, $k_{6}=0.001 \mathrm{~s}^{-1}(\mathrm{x}), k_{5}=1 \mathrm{M}^{-1} \mathrm{~s}^{-1}, k_{6}=1 \mathrm{~s}^{-1}, k_{6}=0.01 \mathrm{~s}^{-1}(\mathrm{y})$ and $k_{5}=50 \mathrm{M}^{-1} \mathrm{~s}^{-1}, k_{6}=1 \mathrm{~s}^{-1}, k_{6}=0.01 \mathrm{~s}^{-1}(\mathrm{z})$, with $k_{1}=50 \mathrm{M}^{-1} \mathrm{~s}^{-1}, k_{1}=k_{-5}=2500 \mathrm{~s}^{-1}, k_{2}=750 \mathrm{~s}^{-1}, k_{2}=75 \mathrm{~s}^{-1}, k_{3}=2500 \mathrm{~s}^{-1}, k_{3}=200 \mathrm{M}^{-1} \mathrm{~s}^{-1}$, $k_{4}=250000 \mathrm{M}^{-1} \mathrm{~s}^{-1}, k_{-4}=25000 \mathrm{M}^{-1} \mathrm{~s}^{-1}$. 


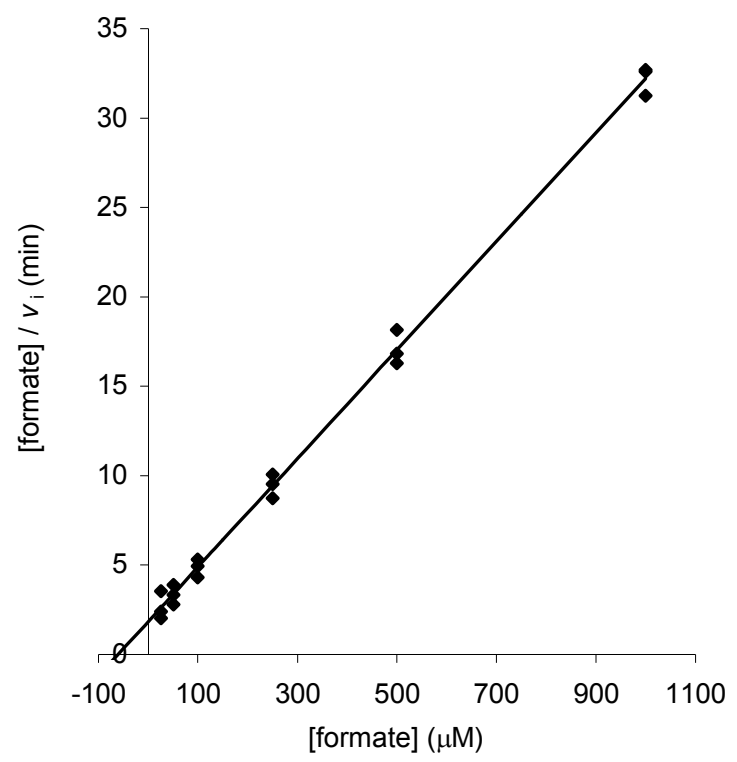

Figure S3. Hanes plot for the determination of the kinetic parameters of Dd FDH for formate oxidation. 


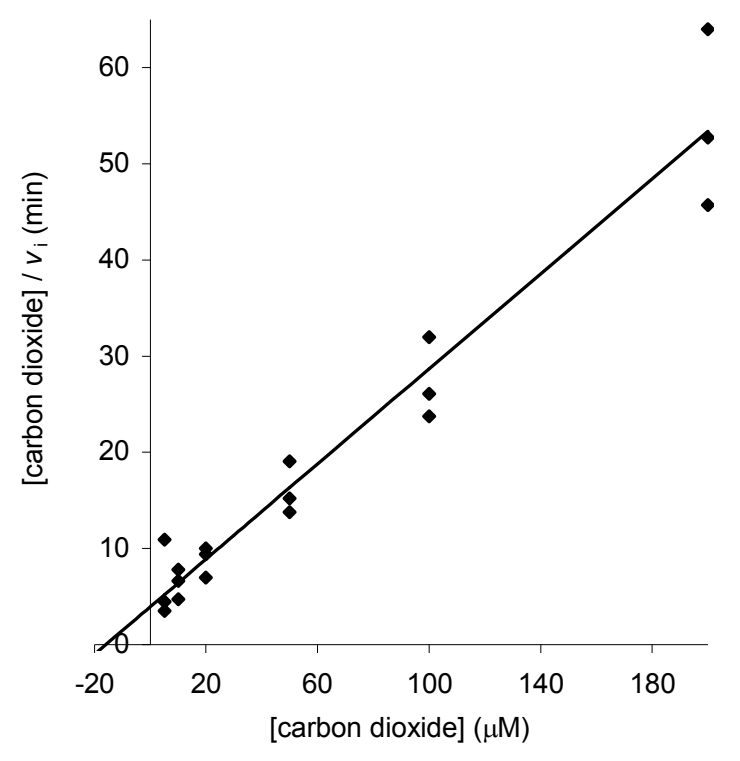

Figure S4. Hanes plot for the determination of the kinetic parameters of Dd FDH for carbon dioxide reduction. 


\section{S2. Kinetic model for the FDH-catalyzed formate oxidation and carbon dioxide reduction}

The kinetic model depicted in figure 4 is suggested to operate as follows.

(a) Catalysis of formate oxidation (blue lines): The initial step is the formate (A) binding to the oxidized $F$ form $\left(F_{0}\right)$ to yield the $F_{0} A$ complex (the subscript " 0 " denotes oxidized molybdenum center; blue dashed lines). This complex can, then, "isomerise" to give an $\mathrm{E}_{0} \mathrm{~A}$ complex, in a slow reaction $\left(k_{6}\right)$ comparatively to the other reactions $\left(k_{1}, k_{2}, k_{3}\right.$ and $k_{4}$; blue lines). The reverse conversion of $\mathrm{E}_{0} \mathrm{~A}$ into $\mathrm{F}_{0} \mathrm{~A}$ is suggested to be kinetically unimportant $\left(k_{6}<<k_{2}\right)$. The $\mathrm{E}_{0} \mathrm{~A}$ complex is catalytically competent and, through the faster $k_{1} \rightarrow k_{2} \rightarrow k_{3} \rightarrow k_{4}$ cycle, it can produce carbon dioxide (P) and reduced enzyme in the $\mathrm{E}$ form

( $E_{2} ;$ the subscript "2" denotes two-electron reduced molybdenum center). The reduced $E_{2}$ form is subsequently oxidized by the artificial electron acceptor, viologen (B), to yield the oxidized kinetically active $E_{0}$ enzyme form, which can start directly a new catalytic cycle. In this way: (i) initially, the concentration of $\mathrm{F}_{0} \mathrm{~A}$ is high and the formation of carbon dioxide and reduced viologen is limited by the slow conversion $\left(k_{6}\right)$ of $\mathrm{F}_{0} \mathrm{~A}$ into the catalytic active $\mathrm{E}_{0} \mathrm{~A}$ complex, leading to an initial lag phase with a very slow viologen reduction; (ii) at latter times, after some catalytic cycles, the kinetically active $E$ form builds up and continues the catalysis through the faster $k_{1} \rightarrow k_{2} \rightarrow k_{3} \rightarrow k_{4}$ cycle, circumventing the slow conversion of $\mathrm{F}$ into $\mathrm{E}$ and leading to the subsequent faster phase of viologen reduction.

To accommodate the "reduced viologen activation", two additional steps were added to the kinetic model (red dashed lines). The first one is the reduction of the inactive $F$ form $\left(k_{-7}\right)$ by reduced viologen $(\mathrm{Q})$ and the second involves the "isomerisation" of $F$ to give the catalytically competent reduced $E_{2}$ form, in a slow reaction $\left(k_{8}\right)$. As for $F_{0} A / E_{0} A$, we suggest that the reverse conversion of $E_{2}$ into $F_{2}$ is kinetically unimportant $\left(k_{8}<<k_{4}\right)$. Subsequently, because we have no evidence that formate can react with reduced $\mathrm{FDH}$, we suggest that $\mathrm{E}_{2}$ is oxidized by oxidized viologen $\left(k_{4}\right)$, yielding the $E_{0}$ form that can initiate the catalytic cycle. After some catalytic cycles, the kinetically active E form is accumulated and, as described above, it can continue the catalysis, circumventing the slow steps of $\mathrm{F}$ into $\mathrm{E}$ conversion. Being parallel to the "formate activation" model (blue dashed lines), this model of "reduced viologen activation" (red dashed lines) can also generate sigmoidal time courses. Overall, the "formate activation" steps ( $k_{5}$ plus $k_{6}$ ) are suggested to be slower than the "reduced viologen activation" steps $\left(k_{7}\right.$ plus $\left.k_{8}\right)$, in order to model the longer lag phases observed with the "formate activation" process.

(b) Catalysis of carbon dioxide reduction (red lines): The inactive $F_{0}$ form is converted into $E_{2}\left(k_{7}, k_{8}\right.$; red dashed lines) as described above. The reverse conversion of $E_{2}$ into $F_{2}$ is suggested to be kinetically unimportant $\left(k_{8}<<k_{-}\right)$. Subsequently, $E_{2}$ reacts with carbon dioxide to produce formate and oxidized $E_{0}$, which, after being reduced by reduced viologen, can start "directly" a new catalytic cycle. Therefore, in parallel to what was described above: (i) initially, the concentration of $F_{2}$ is high and the formation of formate and oxidized viologen is limited by the slow conversion $\left(k_{-8}\right)$ of $F_{2}$ into the catalytic active $E_{2}$, leading to an initial lag phase with a very slow viologen oxidation; (ii) at latter times, after some catalytic 
cycles, the kinetically active $\mathrm{E}$ form builds up and continues the catalysis through the faster $k_{-4} \rightarrow k_{-3} \rightarrow$ $k_{2} \rightarrow k_{1}$ cycle, circumventing the slow conversion of $\mathrm{F}$ into $\mathrm{E}$ and leading to the subsequent faster phase of viologen oxidation. 


\section{S3. FDH reductive activation}

The necessity of one activation process, that is reductive in nature, to obtain high catalytic rates (sections 3.1., 3.2., 3.4. and 3.5.) and to inactivate the enzyme with cyanide (section 3.6), is clear from the results described in this work. The rationalization of that reductive activation process at the molecular/atomic level is, however, particularly challenging with the present knowledge of FDHs. Nonetheless, some hypotheses are envisaged.

(These hypotheses would apply to Mo-FDHs and W-FDHs and to enzymes harboring and active site selenocysteine or cysteine residue ${ }^{27}$, but, for simplicity, they will be here described for a selenocysteinecontaining Mo-FDH.)

Hypothesis (i) - To be active, FDH needs to change the first coordination sphere of the molybdenum atom to become penta-coordinated

The FDH reduction could trigger a conformational alteration that would be responsible for changing the first coordination sphere of the molybdenum atom, leading to the dissociation of the selenocysteine residue and formation of a penta-coordinated center. Although the result of this alteration would be equal to that of the sulfur shift mechanism (this mechanism (discussed in section S4) suggests that formate specifically triggers the selenocysteine dissociation), the way to achieve that outcome would be necessarily different: it would be via a reduction event and not specific formate/active site interaction.

A parallel reductive activation mechanism was described for cytochrome $c$ peroxidase ${ }^{\mathrm{S} 1}$ and cytochrome $c$ and $d_{1}$-containing nitrite reductase ${ }^{\mathrm{S2}}$. Both enzymes harbor one electron transfer heme plus one catalytic heme; the enzymes reduction is essential to trigger a concerted polypeptide movement that changes the hemes coordination sphere, converting the inactive hexa-coordinated catalytic heme into a penta-coordinated active center, ready to react with its substrate. A similar concerted polypeptide movement could be responsible for the selenocysteine dissociation from the molybdenum center of FDH.

Hypothesis (ii) - To be active, FDH needs to change the second coordination sphere of the molybdenum atom

Alternatively, the FDH reduction could trigger a conformational alteration that would be responsible for changing only the second coordination sphere of the molybdenum center. Small changes of the metal center geometry, imposed by the alteration of the protein environment, could alter the energy level of the metal $d$ orbitals, in particular of the $d_{x y}$ orbital in the ground state, thus modulating the reduction potential of the molybdenum center. ${ }^{\mathrm{S3}}$ The influence of the protein can be clearly envisaged when the octahedral geometry found in model compounds is compared with the trigonal prismatic coordination geometry observed in the Mo/W-bis PGD enzyme family. ${ }^{\mathrm{S4}}$

Another possibility is that the alteration in the second coordination sphere of the molybdenum center could be responsible for modifying the substrate binding pocket. That conformational alteration would, e.g., convert an "inoperative" site into a binding site with the correct configuration to bind "correctly" the 
substrate.

Hypothesis (iii) - To be active, FDH needs to change the pyranopterin molecules that coordinate the molybdenum atom

A third possible scenario concerns the pyranopterin molecules that coordinate the molybdenum atom (Fig. 1.C). It was recently suggested that the as prepared periplasmatic nitrate reductase from Rhodobacter sphaeroides holds its proximal pyranopterin cofactor molecule in a fully oxidized and ring-opened form; this enzyme form was suggested to need an activation mechanism, that involves both the cyclisation of the pyran ring and the reduction of the pterin to yield a catalytically competent tricyclic tetrahydropyranopterin cofactor. ${ }^{19 a}$ Also the $R$. sphaeroides dimethylsulfoxide reductase was suggested to need a reductive activation to promote the correct coordination of its $\mathrm{Q}$ pyranopterin cofactor. ${ }^{19 \mathrm{~b}}$

With the currently available knowledge, all these hypotheses seem plausible and we presently have no experimental evidence about the conformational change(s) that would trigger the activation of FDH or about which redox center, or centers, need to be reduced in order to elicit the activation. Nevertheless, other enzymes of the Mo/W-bis PGD enzyme family were described to also need a reductive activation, including nitrate reductases from $R$. sphaeroides ${ }^{19 a, 20 a}, E$. coli ${ }^{20 b}$, Paracoccus pantotrophus ${ }^{20 c}$ and dimethylsulfoxide reductase from $R$. sphaeroides ${ }^{19 b, 20 d}$, which suggests that the reductive activation could be a common phenomenon to this enzyme family. 


\section{The sulfur shift mechanism}

(The FDH reaction mechanism is believed to be similar in Mo-FDHs and W-FDHs and in enzymes harboring and active site selenocysteine or cysteine residue ${ }^{27}$, but, for simplicity, it will be here describe for a selenocysteine-containing Mo-FDH.)

Extended DFT calculations carried out by our group and collaborators led to the comprehensive "sulfur shift mechanism". 15,30

In general terms, the sulfur shift mechanism can be divided into three main stages (Fig. S5). (a) In an initial activation stage, the molybdenum center, that is initially hexa-coordinated, becomes penta-coordinated through the dissociation of the selenocysteine residue from the molybdenum atom (Fig. S5 (i) $\rightarrow$ (ii)). It is suggested that when formate approaches the molybdenum center, with its oxygen moving toward the sulfo group $(\mathrm{Mo}=\mathrm{S})$, it pushes the sulfur to the position originally occupied by the selenocysteine residue, to yield a (Cys)Se-S-Mo bond. In this process, the metal is formally reduced to $\mathrm{Mo}^{4+}$ (intramolecular reduction at the expenses of making a persulfide Se/S-S bond). A vacant coordination position is, thus, created in the metal coordination sphere, to which formate binds, to form a reduced $\mathrm{Mo}^{4+}-\mathrm{OCO}(\mathrm{H})$ complex (Fig. S5 (ii)). (b) In the catalytic part, the (Cys)Se-S-Mo bond is cleaved and the uncoordinated selenocysteine residue, $-(\mathrm{Cys}) \mathrm{Se}^{-}$, stabilized by a hydrogen bond with the conserved histidine, abstracts the formate $\mathrm{C} \alpha$ proton (to yield -(Cys)SeH) (Fig. S5 (iii) $\rightarrow$ (iv)). A carbon dioxide moiety is formed attached to the molybdenum (Mo-S- $\left.\mathrm{CO}_{2}\right)$. Carbon dioxide is eventually released and a penta-coordinated reduced center is formed, with the sulfo group as the fifth axial ligand, $\mathrm{Mo}^{4+}=\mathrm{S}$ (Fig. S5 (v)), in accordance with the X-ray data interpretation of Raaijmakers and Romão ${ }^{11}$. (c) In a final stage, the catalytic cycle is closed, with the oxidation of $\mathrm{Mo}^{4+}$ to $\mathrm{Mo}^{6+}$, via intramolecular electron transfer to other(s) redox center(s), and deprotonation of the selenocysteine residue (Fig. S5 $(v) \rightarrow(v i))$. The molybdenum centre can, then, bind a new formate molecule and start a new catalytic cycle. Calculations showed that the catalysis of the second, and following, formate molecules have a lower activation energy than the first activation cycle. In the absence of formate, the selenocysteine-containing loop is reoriented, the (Cys)Se-S-Mo bond reformed and the enzyme returns to the inactive hexacoordinated form (Fig. S5 (i)).

Accordingly to the sulfur shift mechanism ${ }^{15,30}$, the conserved positively charged arginine plays a key role in driving the formate anion into the active site and its subsequent binding to the molybdenum in the correct position; it would also play a role in product release. The conserved histidine plays a key role in stabilizing, through a hydrogen bond, the uncoordinated selenocysteine (essential to facilitate the formate $\mathrm{C} \alpha$ proton abstraction by the selenocysteine). The terminal sulfo group, $\mathrm{Mo}=\mathrm{S}$, however, does not play any catalytic role in the sulfur shift mechanism, being suggested to only contribute to "anchor" transiently the carbon dioxide moiety to the molybdenum, via a Mo-S- $\mathrm{CO}_{2}$ complex. 


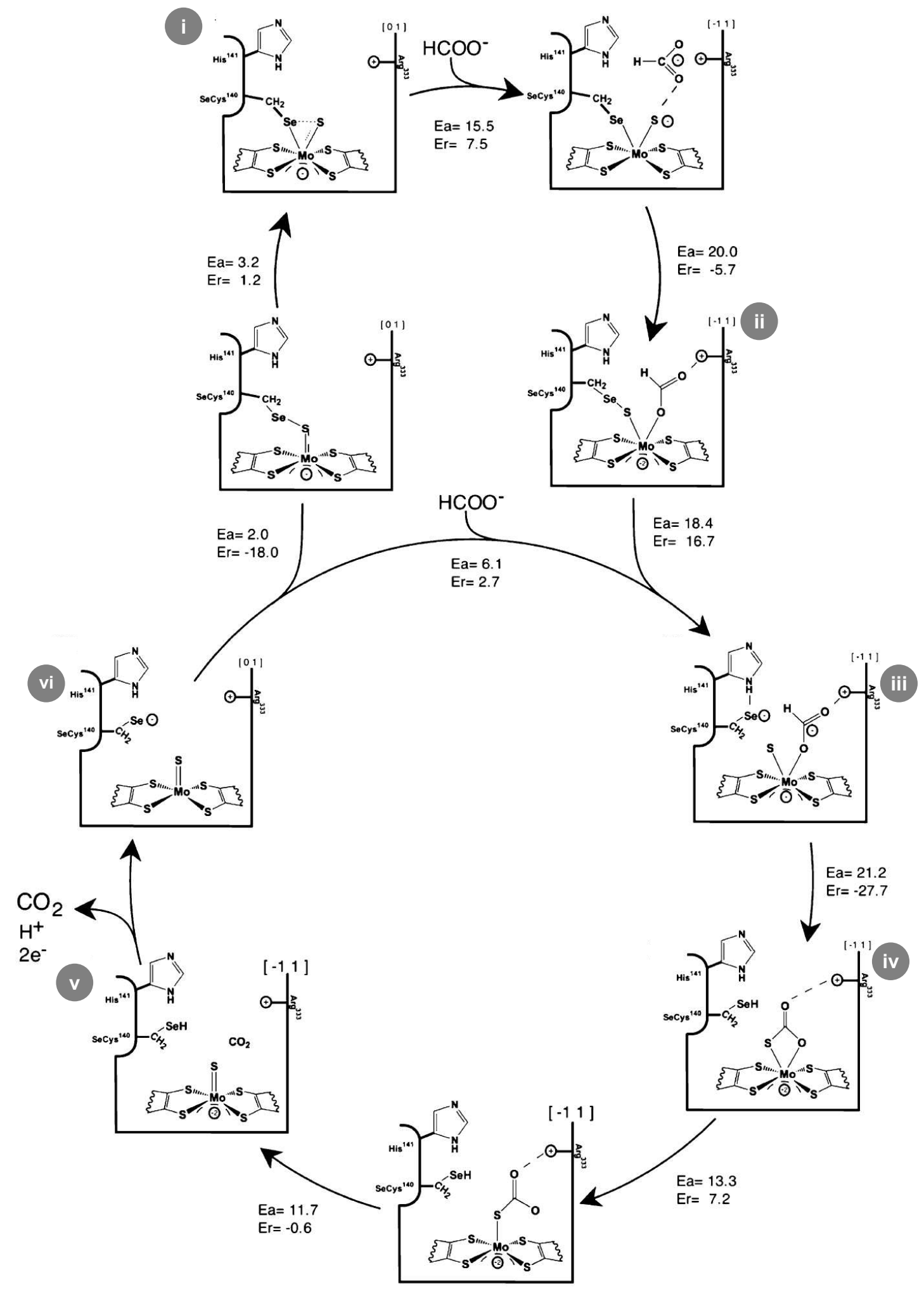

Figure S5. The sulfur shift mechanism proposed for FDH-catalyzed formate oxidation, as suggested by Mota et al. ${ }^{15}$. See text for details. The $E_{\mathrm{a}}$ and $E_{\mathrm{r}}$ values are expressed in $\mathrm{kcal} / \mathrm{mol}$. Adapted with permission from ${ }^{15}$. 
This mechanistic suggestion came following the previous sulfur shift mechanism proposed by our group and collaborators for the periplasmatic nitrate reductase from D. desulfuricans and Cupriavidus necator. ${ }^{\mathrm{S5}, \mathrm{S7}}$ The molybdenum center of those periplasmatic nitrate reductases shares the hexa-coordination depicted in figure 1.A, but with a cysteine residue bound to the molybdenum atom (instead of the selenocysteine depicted there). In these enzymes, the creation of a vacant coordination position is essential for nitrate binding through one of its oxygen atoms, $\mathrm{Mo}^{4+}-\mathrm{ONO}_{2}(-\mathrm{S}-\mathrm{S}(\mathrm{Cys}))$. It is that oxygen atom that will be, subsequently, abstracted by the enzyme active site, to yield nitrite and a $\mathrm{Mo}^{6+}=\mathrm{O}(-\mathrm{S}-\mathrm{S}(\mathrm{Cys})$ ) center (eq. i), in a "classic" oxygen atom transfer reaction. To create the vacant position, it was originally suggested the sulfur shift mechanism. ${ }^{57}$ Hence, because both FDH and periplasmatic nitrate reductases hold identical molybdenum center structures, a similar mechanism, involving a sulfur-shifting, was proposed also for FDH.

$$
\mathrm{ONO}_{2}^{-}+2 \mathrm{H}^{+}+2 \mathrm{e}^{-} \rightleftharpoons \mathrm{NO}_{2}^{-}+\mathrm{H}_{2} \mathrm{O}
$$

Nevertheless, it should be noted that, while nitrate reductases catalyze a "classic" oxygen atom transfer reaction, as is characteristic of almost all molybdenum- and tungsten-containing enzymes ${ }^{6 c, 9}$, the FDH reaction is not an oxygen atom transfer reaction (eq. 1 or 3 versus eq. 2). 


\section{S5. Selenocysteine dissociation from the FDH molybdenum center and formate binding to molybdenum}

As far as we know, presently, there is no unambiguous experimental evidence that formate (or carbon dioxide) binds directly to the FDH molybdenum atom, nor there is experimental consensus regarding the selenocysteine dissociation from the FDH molybdenum atom in the reduced state.

(The discussion below refers to Mo-FDHs and W-FDHs and to enzymes harboring and active site selenocysteine or cysteine residue ${ }^{27}$, but, for simplicity, whenever possible, only a selenocysteinecontaining Mo-FDH will be referred.)

\section{S5.1. Selenocysteine dissociation from the FDH molybdenum center}

One controversial key aspect of the FDH structure concerns the molybdenum coordination sphere in the reduced state, for which, presently, there is no experimental consensus. Raaijmakers and Romão ${ }^{11}$, in a reinterpretation of the original data of Boyington et al. ${ }^{10}$, described that the $E$. coli FDH $\mathrm{H}$ molybdenum center, in the reduced state, has the selenocysteine residue shifted away (12A) from the molybdenum atom (Fig. S1.C; Fig. 1.B - structure on the right). However, presently, there are several lines of evidence suggesting that the FDHs active site selenocysteine/cysteine residue does not become dissociated from the molybdenum/tungsten atom after the enzyme reduction (Fig. 1.B - structure on the left).

All the presently available FDH crystal structures, with the exception of the work of Raaijmakers and Romão ${ }^{11}$, were interpreted as showing the selenocysteine residue bound to the molybdenum ${ }^{10,12}$ or tungsten $^{8 f}$ atom (Fig. S1.A,B,D,E). Moreover, X-ray absorption spectroscopy (XAS) results obtained with FDHs from $E$. coli, $R$. capsulatus and also Dd FDH itself suggest that both the oxidized and reduced molybdenum center hold the selenocysteine/cysteine bound to the molybdenum atom: the $E$. coli enzyme EXAFS data at both the Mo and Se K-edges were interpreted as indicating the presence of four or five Mo-S bonds of $2.36 \AA$, one Mo-Se bond of $2.62 \AA$, plus one Se-S bond of $2.19 \AA$ (between the sulfo group and the selenocysteine selenium) ${ }^{58 a}$; a very similar selenium coordination was suggested for the $D$. desulfuricans $\mathrm{FDH}^{\mathrm{Ssb}}$; one Mo-S(Cys) bond of $2.63 \AA$ was suggested for the $R$. capsulatus FDH (that is an active site cysteine-containing enzyme) ${ }^{31}$. Further experimental evidence came from electron paramagnetic resonance (EPR) spectroscopy that clearly showed that the selenocysteine/cysteine must remain bound to the $\mathrm{Mo}^{5+}$ center of formate-reduced enzyme. ${ }^{\mathrm{S} 9}$ (i) When the EPR spectrum is obtained from ${ }^{77}$ Se-enriched enzyme, a very strong and anisotropic interaction with selenium is observed $\left(A_{1,2,3}{ }^{77} \mathrm{Se}\right)=13.2,75,240 \mathrm{MHz}{ }^{26}$, which is almost five times higher than the one observed in Mo-Se model compounds ${ }^{26,510}$ ). This interaction and the observation of the expected ${ }^{95,97}$ Mo hyperfine coupling confirm that the selenium atom of the selenocysteine is directly coordinated to the $\mathrm{Mo}^{5+}$ (with the unpaired electron delocalized $17-27 \%$ over the selenium) and excludes the possibility of the EPR 
signal arising from a selenium-centered radical. ${ }^{26}$ (ii) Also the hydrogen atoms of the $\beta$-methylene carbon of the selenocysteine residue are thought to be in the close proximity of the molybdenum atom, being responsible for an interaction with a not solvent-exchangeable protons $\left(A_{1}=35.1 \mathrm{MHz}\right){ }^{8 \mathrm{i}}$ (iii) Photolysis assays additionally confirmed that the selenium/sulfur ligation is retained in the FDH $\mathrm{Mo}^{5+}$ center (because the light beam did not affect the ${ }^{77} \mathrm{Se}$ interaction). ${ }^{26}$ (iv) The $\mathrm{Mo}^{5+}$ hexa-coordination (resulting from having the selenocysteine/cysteine residue bound to the molybdenum atom) was also supported by theoretical calculations on the signals-giving species of FDHs. ${ }^{36}$ A different type of evidence was given by the very recent demonstration that the FDH activity is not affected by the iodoacetamide treatment (at $\mathrm{pH} 6$ to 10). ${ }^{\mathrm{S} 11}$ This alkylating agent reacts with "free" ionized selenocysteine and cysteine residues (carboxamidomethylation) and the absence of effect on FDH suggests that the active site selenocysteine/cysteine residue is bound to the molybdenum and not "free" to react with the iodoacetamide.

Hence, it is evident to us that additional experimental work is needed to solve the structural ambiguities of the FDHs active site. It is possible that the crystallization/irradiation had induced some artifacts that are not relevant to the enzyme activity; but it is also possible that the species crystallographically characterized, being catalytically relevant, bear no relation to the species observed by XAS and EPR. Certainly, high resolution structures are needed to confirm the existence of the two alternating conformations of the selenocysteine (cysteine)-containing polypeptide loop and to discuss the catalytic relevance of each conformation.

\section{S5.2. Formate binding to the molybdenum atom}

There is also no clear-cut evidence that formate, or carbon dioxide, binds directly to the FDH molybdenum atom. (i) There is no crystal structure showing the formate molecule in the active site; only Boyington et al. ${ }^{10}$ described the structure of FDH treated with the inhibitor nitrite, which shows the selenocysteine bound to the molybdenum atom and the nitrite molecule with one of its oxygen atoms at $2.58 \AA$ from the molybdenum. (ii) The EPR signals obtained with different FDHs reduced with formate ${ }^{26,32}$, including Dd $\mathrm{FDH}{ }^{8 i}$, do not arise from $\mathrm{FDH}$-formate complexes. ${ }^{8 i, 26,32}$ (iii) There is no evidence from other spectroscopic methods showing the binding of formate to molybdenum, except for the recent XAS at the Mo K-edge study of Schrapers et al. ${ }^{31}$. These authors suggested that, in the presence of formate, the cysteine ligation is replaced by a long Mo-O bond of $2.2 \AA$, which they interpreted as arising from the $\mathrm{Mo}-\mathrm{OCO}(\mathrm{H})$ complex. However, the observation of unidentified Mo-X bonds and of further Mo-O bonds in formate-reacted FDH might question that assignment.

The present absence of more definitive experimental evidence and the new results described in this work (and by others), together with a careful reanalysis of our published experimental data on FDHs, led us to 
search for alternative chemical strategies for the FDH-catalyzed reaction and, thus, to support a mechanism that does not involve the necessity of dissociating the selenocysteine (cysteine) residue nor the formate binding to the molybdenum (tungsten) atom. 


\section{S6. EPR spectroscopic studies of FDH}

The proposed role for the sulfo group in catalysis is in complete agreement with our previous EPR spectroscopic studies with $\mathrm{Dd} \mathrm{FDH}^{8 \mathrm{i}}$ that showed that the formate $\mathrm{C} \alpha$ hydrogen atom is transferred to an acceptor group located within magnetic contact to the molybdenum atom. Formate-reduced Dd FDH originates an $\mathrm{Mo}^{5+}$ EPR signal $\left(g_{1,2,3}=2.012,1.996,1.985\right)$ that shows hyperfine coupling with one solvent-exchangeable proton $\left(A_{1,2,3}=23.1,29.9,27.8 \mathrm{MHz}\right)$ and one not solvent-exchangeable proton $\left(A_{1}=35.1 \mathrm{MHz}\right){ }^{8 i}$ Those two protons are assigned, respectively, to the sulfo ligand and to the hydrogen atoms of the $\beta$-methylene carbon of the selenocysteine residue that remains bound to the molybdenum atom. Assays with ${ }^{2} \mathrm{H}$-labeled formate (aimed to identify if the formate $\mathrm{C} \alpha$ hydrogen is responsible for that coupling) were inconclusive, because the ${ }^{2} \mathrm{H} /{ }^{1} \mathrm{H}$ exchange with the solvent is faster than the timescale of the "freezing technique" used. However, in the presence of the inhibitor azide, the reaction is decelerated and, using ${ }^{1} \mathrm{H}$ - and ${ }^{2} \mathrm{H}$-labeled formate, it was clearly shown that the formate $\mathrm{C} \alpha$ hydrogen is transferred, within $5 \mathrm{~s}$, to a ligand of the molybdenum atom $\left(A_{1,2,3}=\right.$ (not determined), $\left.21.0,21.1 \mathrm{MHz}\right)$ and, then, exchanged rapidly with the solvent. ${ }^{8 i}$ So, the strongly coupled proton is substrate-derived and, then, solvent-exchangeable.

Similar EPR studies were also recently employed by Niks et al. ${ }^{32}$ to demonstrate that also $R$. eutropha FDH catalyzes the direct transfer of the formate $\mathrm{C} \alpha$ hydrogen to one molybdenum ligand. In parallel to what was described above for the $\mathrm{Dd} \mathrm{FDH}$, the $R$. eutropha FDH Mo ${ }^{5+}$ EPR signal obtained shortly after the addition of ${ }^{2} \mathrm{H}$-labeled formate displays no hyperfine coupling; however, after $1 \mathrm{~min}$ of reaction, the proton coupling increases to give rise to an EPR signal indistinguishable from that obtained with ${ }^{1} \mathrm{H}$-formate and with features similar to the ones of the $\mathrm{Dd} \mathrm{FDH}\left(g_{1,2,3}=2.009,2.001,1.992 ; A_{1,2,3}=18,21\right.$, $18 \mathrm{MHz})^{32}$. As above, the results obtained with $R$. eutropha FDH were interpreted as demonstrating the

formate $\mathrm{C} \alpha$ hydrogen transfer to a solvent exchangeable site in the molybdenum center. ${ }^{32}$

The magnitude of the hyperfine constant of the formate-derived solvent-exchangeable proton $(20-30 \mathrm{MHz})$ is consistent with the hydrogen atom being transferred to a ligand in the first coordination sphere of the molybdenum atom; ${ }^{S 9}$ it is not consistent with hydrogen atom transfer to a dissociated selenocysteine that is found $12 \AA$ or $9 \AA$ apart from the molybdenum atom (distances described for the crystal structure ${ }^{11}$ and optimized theoretical structure ${ }^{15}$ ). Photolysis assays (mentioned above in section S5) additionally support that the selenocysteine/cysteine is not the hydrogen acceptor: while the light beam did not affect the ${ }^{77} \mathrm{Se}$ interaction, it induced the photolysis of the solvent-exchangeable proton ${ }^{26}$, indicating that the selenocysteine/cysteine ligation is retained in the $\mathrm{FDH} \mathrm{Mo}{ }^{5+}$ center, but it is not that residue that binds the strongly coupled proton. Moreover, similar hyperfine constant values were determined in xanthine oxidase, where the strongly coupled proton is originated from the xanthine C8 hydrogen atom (the position that is hydroxylated by that enzyme). ${ }^{\mathrm{S12}}$ 
These EPR studies, obtained with different FDHs, indicate that, if the selenocysteine is dissociated from the molybdenum during catalysis, it is not the hydrogen atom acceptor; if the selenocysteine remains bound, it can not accept the hydrogen atom ${ }^{34}$. In either case, the only molybdenum ligand that can accept the formate $\mathrm{C} \alpha$ hydrogen is the sulfo group. Therefore, because it is not reasonable that the hydrogen is first transferred to the selenocysteine and subsequently to the sulfo group, we interpret these EPR results as clearly supporting that the hydride from the formate molecule is transferred to the sulfo group and, then, eventually, to the solvent. 


\section{S7. Rate Equations}

Global rate equation:

$v=\frac{\partial[\mathrm{Q}]}{\partial \mathrm{t}}=[\mathrm{F}] \frac{w_{13}[\mathrm{~A}][\mathrm{B}][\mathrm{Q}]+w_{4}[\mathrm{~B}][\mathrm{Q}]^{2}+w_{1}[\mathrm{~A}]^{2}[\mathrm{~B}]+w_{2}[\mathrm{~A}]^{2}[\mathrm{~B}]^{2}-w_{14}[\mathrm{~A}][\mathrm{B}][\mathrm{P}][\mathrm{Q}]-w_{15}[A][\mathrm{P}][Q]-w_{9}[P][Q]^{2}}{z}$

where A, B, P and Q represent formate, oxidized viologen, carbon dioxide and reduced viologen, respectively, $W_{i}$ are constants, discriminated below, that include the individual $k_{i}$ (Fig. 4)

$$
\begin{gathered}
\text { and } z=w_{3}[\mathrm{~A}]^{2}+w_{8}[\mathrm{~A}][\mathrm{B}]+w_{16}[\mathrm{~A}][\mathrm{P}]+w_{17}[\mathrm{~A}][\mathrm{Q}]+w_{18}[\mathrm{~B}][\mathrm{Q}]+w_{11}[\mathrm{P}][\mathrm{Q}]+w_{12}[\mathrm{Q}]^{2}+ \\
+w_{7}[\mathrm{~A}]^{2}[\mathrm{~B}]+w_{5}[\mathrm{~A}][\mathrm{B}]^{2}+w_{19}[\mathrm{~A}][\mathrm{B}][\mathrm{Q}]+w_{20}[\mathrm{~A}][\mathrm{B}][\mathrm{P}]+w_{21}[\mathrm{~A}]^{2}[\mathrm{P}]+w_{22}[\mathrm{~A}][\mathrm{P}][\mathrm{Q}]+w_{10}[\mathrm{P}][\mathrm{Q}]^{2}+ \\
+w_{23}[\mathrm{~B}][\mathrm{P}][\mathrm{Q}]+w_{24}[\mathrm{~A}][\mathrm{B}][\mathrm{P}][\mathrm{Q}]+w_{25}[\mathrm{~A}]^{2}[\mathrm{~B}][\mathrm{P}]+w_{6}[\mathrm{~A}]^{2}[\mathrm{~B}]^{2}
\end{gathered}
$$

Rate equation for formate oxidation in the absence of initial carbon dioxide and presence of a constant oxidized viologen concentration:

$$
\begin{gathered}
v=\frac{\partial[\mathrm{Q}]}{\partial \mathrm{t}}=[\mathrm{F}] \frac{k_{\mathrm{cat}}{ }^{\mathrm{app}, \mathrm{A}}[\mathrm{A}]}{K_{\mathrm{m}}{ }^{\mathrm{app}, \mathrm{A}}[\mathrm{A}]} \\
k_{\mathrm{cat}}^{\mathrm{app}, \mathrm{A}}=\frac{w_{1}[\mathrm{~B}]+w_{2}[\mathrm{~B}]^{2}}{w_{3}+w_{7}[\mathrm{~B}]+w_{6}[\mathrm{~B}]^{2}}, \quad K_{\mathrm{m}}{ }^{\mathrm{app}, \mathrm{A}}=\frac{w_{8}[\mathrm{~B}]+w_{5}[\mathrm{~B}]^{2}}{w_{3}+w_{7}[\mathrm{~B}]+w_{6}[\mathrm{~B}]^{2}}
\end{gathered}
$$

Rate equation for carbon dioxide reduction in the absence of initial formate and presence of a constant reduced viologen concentration:

$$
\begin{gathered}
v=-\frac{\partial[\mathrm{Q}]}{\partial \mathrm{t}}=[\mathrm{F}] \frac{K_{\text {cat }}^{\mathrm{app}, \mathrm{P}}[\mathrm{P}]}{K_{\mathrm{m}}{ }^{\mathrm{app}, \mathrm{P}}[\mathrm{P}]} \\
k_{\text {cat }}^{\text {app }, \mathrm{P}}=\frac{k_{\text {cat }}[\mathrm{Q}]}{K_{\mathrm{m}}^{\mathrm{Q}}+[\mathrm{Q}]}, \quad K_{\mathrm{m}}^{\mathrm{app}, \mathrm{P}}=\frac{K_{\mathrm{m}}^{\mathrm{P}}[\mathrm{Q}]}{K_{\mathrm{m}}^{\mathrm{Q}}+[\mathrm{Q}]} \\
k_{\text {cat }}=\frac{W_{9}}{W_{10}}, \quad K_{\mathrm{m}}{ }^{\mathrm{P}}=\frac{W_{12}}{W_{10}}, \quad K_{\mathrm{m}}{ }^{\mathrm{Q}}=\frac{W_{11}}{W_{10}}
\end{gathered}
$$


Definition of the $w_{i}$ constants in terms of the individual rate constants $\left(k_{i}\right)$ of the kinetic model presented in figure 4:

$w_{1}: k_{1} k_{2} k_{3} k_{5} k_{6}\left(k_{4} k_{-8}+k_{7} k_{8}\right)$

$w_{2}: k_{1} k_{2} k_{3} k_{4} k_{5} k_{6} k_{7}$

$w_{3}: k_{1} k_{2} k_{3} k_{5} k_{6}\left(k_{8}+k_{-8}\right)$

$w_{4}: k_{-4} k_{5} k_{6} k_{7} k_{8} y_{1}$

$w_{5}: k_{4} k_{5} k_{6} k_{7} y_{1}+k_{1} k_{4} k_{-5} k_{-6} k_{7}\left(k_{-2}+k_{3}\right)$

$w_{6}: k_{1} k_{4} k_{5} k_{7}\left(y_{3}+k_{3} k_{6}+k_{3} k_{-6}\right)$

w: $y_{5}\left(k_{1} k_{4} k_{5} k_{6} k_{-8}+k_{1} k_{5} k_{6} k_{7} k_{8}\right)+\left(k_{-2}+k_{3}\right)\left(k_{1} k_{4} k_{5} k_{-6} k_{-8}+k_{1} k_{5} k_{-6} k_{7} k_{8}\right)+\left(k_{6}+k_{8}\right)\left(k_{1} k_{2} k_{3} k_{5} k_{7}\right)$

$w_{8}: k_{1} k_{7} k_{8} y_{2}+k_{4} k_{5} k_{6} k_{-8} y_{1}+k_{1} k_{4} k_{-5} k_{-6} k_{-8}\left(k_{-2}+k_{3}\right)+k_{-1} k_{5} k_{6} k_{7} k_{8}\left(k_{-2}+k_{3}\right)$

$w_{g}: k_{-2} k_{-3} k_{-4} k_{-} k_{-8}\left(k_{-5} k_{-6}+k_{-1} k_{-5}+k_{-1} k_{6}\right)$

$w_{10}: k_{-3} k_{-4} k_{-7} k_{-8}\left(y_{3}+y_{4}+k_{-1} k_{6}+k_{-1} k_{-5}\right)+k_{-2} k_{-3} k_{-4} k_{-5} k_{-6} k_{-7}$

$w_{11}: k_{-2} k_{-3} k_{-4} k_{-5} k_{-6} k_{-8}+k_{-1} k_{-2} k_{-3} k_{-7} k_{-8}\left(k_{6}+k_{-5}\right)$

$w_{12}: y_{2}\left(k_{-4} k_{-7} k_{8}+k_{-4} k_{-7} k_{-8}\right)+y_{6}\left(k_{-4} k_{6} k_{-7} k_{8}+k_{-4} k_{-5} k_{-7} k_{8}+k_{-4} k_{6} k_{-7} k_{-8}+k_{-4} k_{-5} k_{-7} k_{-8}\right)$

$w_{13}: k_{2} k_{3}\left(k_{1} k_{4} k_{-5} k_{-7} k_{-8}+k_{1} k_{4} k_{6} k_{-7} k_{-8}+k_{-4} k_{5} k_{6} k_{7} k_{8}\right)$

$w_{14}: k_{-1} k_{-2} k_{-3} k_{-4} k_{5} k_{6} k_{7}$

$w_{15}: k_{-2} k_{-3} k_{-8}\left(k_{-1} k_{-4} k_{5} k_{6}+k_{1} k_{-5} k_{-6} k_{-7}\right)$

$w_{16}: k_{-2} k_{-3} k_{-8}\left(k_{-1} k_{5} k_{6}+k_{1} k_{-5} k_{-6}\right)$

$w_{1}: k_{-4} k_{5} k_{6} k_{-8} y_{1}+k_{-4} k_{5} k_{6} k_{8} y_{1}+k_{1} k_{-7} k_{8} y_{2}+k_{1} k_{-7} k_{-8} y_{2}$

$w_{18}:\left(k_{4} k_{-7} k_{-8}\right)\left(y_{2}+k_{6} y_{6}+k_{-5} y_{6}\right)+\left(k_{-4} k_{7} k_{8}\right)\left(y_{2}+k_{6} y_{6}+k_{-5} y_{6}\right)$

$w_{19}: y_{5}\left(k_{1} k_{4} k_{6} k_{-7} k_{-8}+k_{1} k_{4} k_{-5} k_{-7} k_{-8}+k_{-4} k_{5} k_{6} k_{7} k_{8}\right)+y_{1}\left(k_{-4} k_{5} k_{6} k_{7}+k_{-4} k_{5} k_{7} k_{8}\right)+$

$$
+\left(k_{-2}+k_{3}\right)\left(k_{1} k_{4} k_{-6} k_{-7} k_{-8}+k_{1} k_{4} k_{-5} k_{-6} k_{-7}+k_{-4} k_{5} k_{-6} k_{7} k_{8}\right)
$$

$w_{20}: k_{-2} k_{-3} k_{7}\left(k_{-1} k_{5} k_{6}+k_{1} k_{-5} k_{-6}\right)$

$w_{21}: k_{1} k_{-3} k_{5} k_{-8} y_{3}$

$w_{22}: k_{1} k_{-3} k_{-7} k_{-8}\left(y_{3}+y_{4}\right)+k_{1} k_{-2} k_{-3} k_{-5} k_{-6} k_{-7}+k_{-3} k_{-4} k_{5} k_{-8}\left(k_{-1} k_{6}+y_{3}\right)$

$w_{23}: k_{-2} k_{-3} k_{-4} k_{-5} k_{-6} k_{7}$

$w_{24}: k_{-3} k_{-4} k_{5} k_{7}\left(y_{3}+k_{-1} k_{6}\right)$

$w_{25}: k_{1} k_{-3} k_{5} k_{7} y_{3}$

$y_{1}: k_{-1} k_{-2}+k_{-1} k_{3}+k_{2} k_{3}$

$y_{2}: k_{-2} k_{-5} k_{-6}+k_{2} k_{3} k_{-5}+k_{2} k_{3} k_{6}+k_{3} k_{-5} k_{-6}$

$y_{3}: k_{-2} k_{6}+k_{2} k_{6}+k_{-2} k_{-6}$

$y_{4}: k_{-2} k_{-5}+k_{2} k_{-5}+k_{-5} k_{-6}$

$y_{5}: k_{2}+k_{-2}+k_{3}$

$y_{6}: k_{-1} k_{-2}+k_{-1} k_{3}$ 


\section{References}

(S1) (a) Echalier, A.; Goodhew, C.F.; Pettigrew, G.W.; Fulop, V. Structure 2006, 14, 107-117. (b) Echalier, A.; Brittain, T.; Wright, J.; Boycheva, S.; Mortuza, G.B.; Fulop, V.; Watmough, N.J. Biochemistry 2008, 47, 1947-1956.

(c) Moura, I.; Pauleta, S.R.; Moura, J.J.G. J. Biol. Inorg. Chem. 2008, 13, 1185-1195.

(S2) (a) Williams, P.A.; Fülöp, V.; Garman, E.F.; Saunders, N.F.; Ferguson, S.J.; Hajdu, J. Nature 1997, 389, 406412. (b) Nurizzo, D.; Cutruzzolà, F.; Arese, M.; Bourgeois, D.; Brunori, M.; Cambillau, C.; Tegoni, M. Biochemistry 1998, 37, 13987-13996. (c) Nurizzo, D.; Cutruzzolà, F.; Arese, M.; Bourgeois, D.; Brunori, M.; Cambillau, C.; Tegoni, M. J. Biol. Chem. 1999, 274, 14997-15004. (d) Maia, L.; Moura J.J.G. Chem. Rev. 2014, 114, 5273-5357.

(S3) (a) Lorber, C.; Donahue, J.P.; Goddard, C.A.; Nordlander, E.; Holm, R.H. J. Am. Chem. Soc. 1998, 120, 81028112. (b) Lim, B.S.; Sung, K.M.; Holm, R.H. J. Am. Chem. Soc., 2000, 122, 7410-7411. (c) Lim, B.S.; Holm, R.H. J. Am. Chem. Soc. 2001, 123, 1920-1930. (d) McNaughton, R.L.; Lim, R.S.; Knottenbelt, S.Z.; Holm, R.H.; Kirk, M.L. J. Am. Chem. Soc., 2008, 130, 4628-4636.

(S4) (a) Sung, K. M.; Holm, R. H. J. Am. Chem. Soc. 2001, 123, 1931-1943. (b) Sung, K. M.; Holm, R. H. Inorg. Chem. 2001, 40, 4518-4525. (c) Wang, J. J.; Kryatova, O.P.; Rybak-Akimova, E.V.; Holm, R. H. Inorg. Chem. 2004, 43, 8092-8101.

(S5) The $D$. desulfuricans and $C$. necator enzymes harbor an active site that is different from the active site of the $E$. coli and Rhodobacter sphaeroides periplasmatic nitrate reductases. While the $D$. desulfuricans and $C$. necator enzymes hold the molybdenum atom coordinated by a cysteine sulfur atom plus a terminal sulfo group, $\mathrm{Mo}=\mathrm{S}(-\mathrm{S}(\mathrm{Cys}))$, besides the two pyranopterin cofactor molecules, the $E$. coli and $R$. sphaeroides enzymes have one cysteine sulfur atom plus a terminal hydroxyl group, $\mathrm{Mo}-\mathrm{OH}(-\mathrm{S}(\mathrm{Cys})) .{ }^{\mathrm{S} 6}$

(S6) (a) Jepson, B.J.N.; Mohan, S.; Clarke, T.A.; Gates, A.J.; Cole, J.A.; Butler, C.S.; Butt, J.N.; Hemmings, A.M.; Richardson, D. J. J. Biol. Chem., 2007, 282, 6425-6437. (b) Arnoux, P.; Sabaty, M.; Alric, J.; Frangioni, B.; Guigliarelli, B.; Adriano, J. M.; Pignol, D. Nat. Struct. Biol., 2003, 10, 928-934.

(S7) Cerqueira, N.M.; Gonzalez, J.P.; Brondino,C.D.; Romão, M.J.; Romão, C.C.; Moura, I., Moura, J.J. J. Comput. Chem. 2009, 30, 2466-2484.

(S8) (a) George, G.N.; Colangelo, C.M.; Dong, J.; Scott, R.A.; Khangulov, S.V.; Gladyshev, V.N.; Stadtman, T.C. J. Am. Chem. Soc. 1998, 120, 1267-1273. (b) George, G.N.; Costa, C.; Moura. J.J.G.; Moura, I. J. Am. chem. Soc. 1999, 121, 2625-2626.

(S9) Maia, L., Moura, I., Moura, J.J.G., "EPR Spectroscopy on Mononuclear Molybdenum-Containing Enzymes", in "Metalloenzymes/ Metalloproteins EPR", Vol. 33, Biological Magnetic Resonance, Eds. Hanson, G., Berliner, L.J., 2016, Springer Publishers - in press.

(S10) Hanson, G.R.; Wilson, G.L.; Bailey, T.D.; Pilbrow, J.R.; Wedd, A.G. J. Am. Chem. Soc. 1987, 109, 2609-2616.

(S11) Hartmann, T.; Schrapers, P.; Utesch, T.; Nimtz, M.; Rippers, Y.; Dau, H.; Mroginski, M.A.; Haumann, M.; Leimkühler, S. Biochemistry, 2016, 55, 2381-2389.

(S12) (a) Gutteridge, S.; Tanner, S.J.; Bray R.C. Biochem. J. 1978, 175, 869-878. (b) Gutteridge, S.; Tanner, S.J.; Bray, R.C. Biochem. J. 1978, 175, 887-897. (c) Gutteridge, S.; Bray, R.C. Biochem. J. 1980, 189, 615-623. (d) Malthouse, J.P.G.; Gutteridge, S.; Bray, R.C. Biochem. J. 1980, 185, 767-770. 